\title{
Contabilidad estratégica como disciplina para alcanzar ventajas competitivas e impulsar el desarrollo socioeconómico
}

\section{Strategic accounting as a discipline to achieve competitive advantages and promote socio - economic development}

Letty Karina Elizalde Marín. ${ }^{1}$ \& Adriana Margarita Morales Noriega. ${ }^{2}$

Recibido: 10-06-2019 / Revisado: 15-07-2019 /Aceptado: 04-08-2019/ Publicado: 06-09-2019

\begin{abstract}
.
DOI: https://doi.org/10.33262/cienciadigital.v3i3.3.812

This article was focused on analyzing strategic accounting as a discipline to achieve competitive advantages, with this purpose a methodology of documentary type was used, with bibliographic design, different sources were consulted, gathering relevant information on strategic planning, strategic accounting, objectives, elements and its competitive advantages, reading and interpreting the texts to make the analysis and subsequent final reflections of the content. For the phase in which the information is consolidated, a database prepared for this purpose was used, in which the information collected was kept. In the development of the analysis stage of the documents, sources or data, the textual content sheets were elaborated, which helped to manage the data. By establishing that strategic accounting is a fundamental tool, it must be included, taken into account, for all strategic planning. Strategic accounting generates important, confidential data, which, when well analyzed, interpreted, implemented, contribute to the achievement of competitive advantages and socioeconomic development.
\end{abstract}

Keywords: Strategic planning, strategic accounting, competitive advantages.

\footnotetext{
${ }^{1}$ Escuela Superior Politécnica de Chimborazo, Facultad de Administración de Empresas. Riobamba-Ecuador. letty.elizalde@espoch.edu.ec

${ }^{2}$ Escuela Superior Politécnica de Chimborazo, Facultad de Administración de Empresas. Riobamba-Ecuador. adriana.morales@espoch.edu.ec
} 


\section{Resumen}

Este artículo estuvo enfocado en analizar la contabilidad estratégica como disciplina para alcanzar ventajas competitivas e impulsar el desarrollo socio económico, con este propósito fue utilizada una metodología de tipo documental, con diseño bibliográfico, se consultaron diferentes fuentes, recopilando información relevante sobre la planificación estratégica, contabilidad estratégica, objetivos, elementos y sus ventajas competitivas, realizando lectura e interpretación de los textos para hacer el análisis y posteriores reflexiones finales del contenido. Para la fase en la que se consolida la información, se utilizó una base de datos elaborada para tal efecto, en la cual se guardó lo recabado. En el desarrollo de la etapa de análisis de los documentos, fuentes o datos, se procedió a la elaboración de las fichas de contenido textual, lo que ayudó a manejar los datos. Logrando establecer que la contabilidad estratégica, es una herramienta fundamental, debe ser incluida, tomada muy en cuenta, para toda planificación estratégica. La contabilidad estratégica, genera datos importantes, confidenciales, los cuales, al ser bien analizados, interpretados, puestos en ejecución, contribuyen en el logro de ventajas competitivas y en el desarrollo socioeconómico.

Palabras clave: Planificación estratégica, contabilidad estratégica, ventajas competitivas.

\section{Introducción}

El mundo que hoy se conoce y posiblemente el de mañana, está lleno de trasformaciones, muchas de ellas se están dando en este momento y otras se darán en el futuro, no existe ningún elemento conviviendo en el entorno que no esté involucrado en una posible trasformación o evolución, se vive en un hábitat donde se evoluciona constantemente, en un mercado donde cada día que pasa se exige más y mucho más.

En este sentido, las organizaciones cambian, se transforman, fijan objetivos para poder coexistir en un mercado muy exigente, donde normalmente los poderosos terminan con los débiles, en el cual, las empresas establecen planes estratégicos para ser competitivas, sostenerse en sus sectores y posicionarse como la mejor o una de las mejores.

Por ello, todas las áreas o departamentos que forman parte de la estructura organizacional deben transformarse, unirse a esa campaña de cambio, direccionándose hacia esos objetivos planteados por sus directivos, siendo uno de esos departamentos el contable.

Al respecto de lo planteado, el área contable para las organizaciones es un bastión de apoyo, que concatenado con el resto de los departamentos logran ese éxito esperado, por tal motivo, no puede desligarse del proceso de trasformación y direccionamiento hacia la planificación estratégica. 
De allí, la idea de cambio, de una contabilidad tradicional a una contabilidad estratégica, unida con la contabilidad administrativa, financiera y de costos, logrando así una simbiosis, en función de alcanzar esa competitividad. Por ello se hace necesario analizar la contabilidad estratégica como disciplina para alcanzar ventajas competitivas e impulsar el desarrollo socioeconómico.

\section{Metodología}

Este trabajo fue desarrollado bajo la premisa de una investigación de tipo documental, ya que se concentró exclusivamente en la compilación de información de diversas fuentes, examinar diversos documentos escritos referentes a la contabilidad estratégica como disciplina para alcanzar ventajas competitivas e impulsar el desarrollo socioeconómico.

De acuerdo con lo planteado, Arias (2016), sostiene que la investigación documental es un proceso basado en la búsqueda, recuperación, análisis, crítica e interpretación de datos secundarios, es decir, los obtenidos y registrados por otros investigadores en fuentes documentales: impresas, audiovisuales o electrónicas. Como en toda investigación, el propósito de este diseño es el aporte de nuevos conocimientos.

De igual manera, se enmarcó en un diseño bibliográfico, basándose en la exploración metódica del documental recopilado referente a la contabilidad estratégica como disciplina para alcanzar ventajas competitivas, con la finalidad de un análisis respectivo al área.

En este sentido, Palella y Martins (2010), sostienen que el diseño bibliográfico, se fundamenta en la revisión sistemática, rigurosa y profunda del material documental de cualquier clase. Se procura el análisis de los fenómenos o el establecimiento de la relación entre dos o más variables. Cuando se opta por este tipo de estudio, el investigador utiliza documentos, los recolecta, selecciona, analiza y presenta resultados coherentes.

De igual forma, en cuanto a la técnica de selección y evaluación del material disponible, el trabajo se basó en una actividad exploratoria y una segmentación de la documentación existente sobre el tema, a los fines de ubicar la información necesaria para dar respuesta al objetivo planteado. (Pelekais et al, 2015).

Para la fase en la que se consolida la información, fue utilizada una base de datos elaborada para tal efecto, en la que se guardó lo recabado. En el desarrollo de la etapa de análisis de los documentos, fuentes o datos, se procedió a elaborar las fichas de contenido textual, lo cual ayudó a manejar los datos.

En este sentido, la organización de estos contenidos, así como la revisión esquemática, permitió verificar si el proceso de investigación es acertado, en vez de erróneo, valorar el material recopilado, así como la detección de excesos en las ideas transcritas, con el fin de 
organizar y uniformar la investigación de manera eficaz y de constatar que no falten datos esenciales. Tras el proceso anterior, se clasificó el material recopilado y fueron depurados aquellos datos con mayor valor para responder al objetivo de estudio.

\section{Resultados}

Antes de considerar analizar aspectos de la contabilidad estratégica, se toma como premisa el tema de la planificación estratégica, por ser el soporte que apalanca su desarrollo.

\section{Planificación estratégica}

Según Rodríguez (2005), la planeación estratégica es el conjunto de planes integrales de una organización que normarán el comportamiento futuro de la misma. De igual manera, Martínez (2002), establece que la planeación estratégica de hecho es un proceso de tomar decisiones al más alto nivel, deriva su complejidad en las variadas alternativas u opciones estratégicas que se definen para lograr un determinado objetivo a largo plazo

De acuerdo con Beal y Apfelthaler (citados en Quintal 2005), conforme las actividades y áreas funcionales de la organización se tornen más complejas, la planeación estratégica se desarrollará a través de varias fases: comenzará con planes financieros simples y presupuestos; continuará con la planificación basada en pronósticos; posteriormente se llevará a cabo la planificación orientada externamente.

Los autores arriba mencionados, coinciden en que la planeación estratégica es un proceso a largo plazo, el cual consistirá en el avance, ejecución de ciertas metas y el modo en que se deberán desempeñar para llegar al resultado deseado. Sin embargo, la operativa no es estática, debe y puede ser monitoreada a lo largo del tiempo de ejecución a fin de detectar y corregir posibles desviaciones. La planeación estratégica puede ser aplicable a cualquier tipo de organización; sin embargo, se debe tener en cuenta la tropicalización de la misma, dependiendo del negocio de que se trate, de sus características, así como las condiciones.

Por ello, contextualizar el desarrollo de esta herramienta, contribuye al logro de las metas que cualquier organización se fije como alcanzables.

\section{Contabilidad estratégica}

Velastegui (2012), es una disciplina que provee información necesaria para formular, interpretar y llevar acabo estrategias con el fin de alcanzar una ventaja competitiva. Esto adquiere especial relevancia en el ambiente globalizado, cada vez más complejo y competitivo, teniendo en muchos casos el enfrentamiento entre organizaciones.

De acuerdo al autor Arrarte (2012), la contabilidad estratégica, es la disciplina que provee información necesaria para formular y llevar a cabo las estrategias con el fin de alcanzar una ventaja competitiva. Es fundamental, para el proceso de toma de decisiones. Sirve para 
establecer los segmentos de mercados más rentables, definir, asignar los recursos necesarios, los planes de acción a ejecutar para sobresalir ante la competencia y la búsqueda de alianzas para permitirles ser más competitivos.

Así mismo, SUMMA ENTERPRISSE (2016), la define como el sistema de que incluye información financiera y no financiera para la toma de decisiones destinada a soportar, mantener e incrementar la posición competitiva de una organización. La contabilidad estratégica reemplaza a la tradicional, puede mostrar mucho, pero oculta lo más importante, porque las empresas no solo son números sino también toman en cuenta muchos otros factores que la afectan.

Igualmente, Montes. (2009, pág. 45), señala que la contabilidad de direccionamiento estratégico se puede definir como la contabilidad al servicio donde la estrategia es entendida como: "definición de las metas y objetivos básicos a largo plazo en una empresa, junto con la adopción de cursos de acción y distribución de recursos necesarios para lograr estos propósitos"

Al mismo tiempo, Farfán (2010), afirma que la contabilidad de direccionamiento estratégico puede verse como la versión mejorada, adaptada a los cambios del entorno, la contabilidad de gestión, cuyo fin es satisfacer los requerimientos informativos de los usuarios internos a la organización, especialmente del administrador, para preparar y presentar información que sirva para la toma de decisiones estratégicas, permitiendo generar ventajas competitivas en busca de excelencia empresarial, con una visión de más largo plazo, de incursión en nuevos mercados, garantice, además la sostenibilidad en el tiempo, el crecimiento empresarial.

En consecuencia, la contabilidad estratégica, es el resultado de una simbiosis entre diferentes procesos contables, que apoyan significativamente a las organizaciones con el logro de sus objetivos, contribuyendo como respaldo para la toma de decisiones, con un fin último, obtener ganancias en el mercado.

\section{Objetivos de la contabilidad estratégica}

De acuerdo a Farfán (2010), los objetivos de este tipo de contabilidad van ligados a la visión estratégica organizacional, donde se desprenderán decisiones operativas o de gestión, tratarán de coordinar todo tipo de esfuerzos en el seno de una organización en un determinado entorno, entre ellos están:

a) Proveer y analizar información para la formulación de estrategias del negocio: La contabilidad estratégica ayuda al establecimiento de las estrategias al proveer información referente a cadena de valor organizacional. Busca identificar, eliminar las actividades que no agregan valor, e identificar y aprovechar los enlaces entre las 
actividades generadoras de valor a través de colaboraciones entre los departamentos, los clientes y los proveedores.

b) Comunicar la estrategia en la organización mediante el establecimiento de indicadores de medición del desempeño: La contabilidad estratégica reconoce que cada objetivo estratégico debe estar relacionado con los factores críticos del éxito empresarial y con los indicadores de desempeño atados a aquellos. En el conjunto de dichos indicadores deben existir tanto indicadores predictivos como descriptivos evidenciando la situación actual. De esta manera la contabilidad estratégica facilita el desarrollo de una respuesta rápida ante los cambios en el mercado en su ambiente.

c) Monitorear la validez estratégica del negocio: Para que la estrategia sea relevante debe permitir a la compañía alcanzar un equilibrio entre sus fuerzas, debilidades internas, entre sus oportunidades y amenazas externas (FODA). Debido a que la estrategia se enfoca al futuro, la empresa debe hacer supuestos acerca del estado futuro del ambiente, de operaciones tanto externas como internas. Monitorear la realización, su éxito, dando posibilidades a los administradores de validar dicha estrategia, en caso de no ser apropiada para la circunstancia, poder modificarla para que sea relevante y útil para la organización.

Por otra parte, para Lunar (2016), asegura que de los objetivos de la contabilidad estratégica, los cuales van ligados directamente a la visión estratégica organizacional, se desprenderán decisiones operativas o de gestión, que coordinarán los esfuerzos al interior empresarial.

Así mismo, la contabilidad estratégica, como sistema de información integral, se encarga de monitorear los diferentes módulos o áreas, trasmitiendo en tiempo real, la situación o estado del proceso contable. En algunas empresas lo llaman SIG (Sistema de Información Gerencial) base para la toma de decisiones y gestión de actividades. De esa forma se logran los objetivos desprendidos Misión y Visión implantadas por la organización.

Agrega la autora, la contabilidad estratégica garantiza el enfoque hacia la rentabilidad empresarial, también juega el importante papel de medida de desempeño organizacional.

En este sentido, la Contabilidad Estratégica se nutre de la contabilidad financiera, administrativa, y contabilidad de costos, para tener una visión más amplia, dinámica, que le permita adaptarse al entorno, poder adelantarse al futuro para garantizar la supervivencia empresarial. Proporciona información que ayuda a planificar, controlar las operaciones en las organizaciones, va directamente relacionada al ciclo del proceso administrativo: proveer, planear, organizar, implementar, dirigir y controlar.

\section{- Contabilidad financiera}

Para Alcarria (2008), es un área de la contabilidad que tiene como objetivo preparar y elaborar información contable destinada a los usuarios externos. Dicha información está 
sujeta a una regulación externa a la empresa (del estado, de organizaciones profesionales, entre otros.

De acuerdo a Mallo y Pulido (2008), es un sistema de información que permite medir la evolución del patrimonio o riqueza, los resultados o rentas periódicas de la empresa, mediante el registro sistemático de las transacciones realizadas en su actividad económico-financiera, lo conduce a la elaboración de cuentas anuales, preparadas con arreglo a principios contables, normas de valoración uniformes, posibilitando que sean interpretadas y comparadas por los agentes económicos interesados en conocer el funcionamiento empresarial.

\section{- Contabilidad Administrativa}

Robles y Alcerreca (2008), definen la contabilidad administrativa como la información contable generada en una empresa expresada tanto en términos monetarios como no monetarios, para la toma de decisiones de la administración de un ente económico.

Para Horngren et al (2006), la contabilidad administrativa es la rama donde la contabilidad genera información para los directivos de una organización. Consiste en el proceso de identificar, medir, acumular, analizar, preparar, interpretar y comunicar la información que los ayude a cumplir los objetivos organizacionales. En este sentido, cada uno de los aspectos incorporados por esta herramienta va permitir asumir una efectiva toma de decisión.

\section{- Contabilidad de Costos}

Según Horngren et al (2010), "la contabilidad de costos proporciona información para dirigir la contabilidad administrativa, la financiera" (p. 02). La contabilidad de costos mide, analiza, presenta información financiera y no financiera relacionada con los costos de adquirir o utilizar recursos en una organización. De acuerdo al referido autor, la contabilidad de costos es un área de la contabilidad que se ocupa de planeación, clasificación, acumulación, control y asignación de costos. Se conoce también como contabilidad analítica, conforma junto a la contabilidad administrativa y contabilidad financiera, la estructura contable organizacional.

Como se puede apreciar, el objetivo general de la contabilidad estratégica, es el compendio de los procesos contables, en función del manejo de información privilegiada, la misma será registrada, catalogada, descrita y analizada, con el fin de mejorar todos los procesos para el logro de los objetivos planteados.

\section{Elementos de la contabilidad estratégica}

SUMMA ENTERPRISSE (2016), hace referencia que dentro de los elementos la contabilidad estratégica se tiene al: Balanced Scorecard, Valor Económico Agregado (EVA), 
el Costeo ABC, entre otros. En este orden de ideas, se esboza aquello que los contadores deberían saber a grandes rasgos sobre el tema.

Balance Scorecard CMI, es un enfoque de cómo incorporar los objetivos estratégicos en el sistema administrativo a través de los mecanismos de medición del desempeño. El Balance Scorecard traslada la visión y la estrategia, al método que motiva el seguimiento de las metas establecidas al largo plazo.

Valor Económico Agregado - EVA, su objetivo fundamental es maximizar la creación de valor a través del cambio de cultura, para que todos los gerentes y colaboradores piensen, sientan, actúen como si fueran los dueños a la hora de tomar decisiones. Las ganancias contables no son suficientes para una empresa, si no son mayores los costos de oportunidad del capital invertido.

Costos ABC, el modelo de costo $\mathrm{ABC}$ es una guía que se basa en la agrupación en centros de costos que conforman una secuencia de valor de los productos y servicios, generando la actividad productiva. Las actividades causan costos, se relacionan a través de su consumo con el costo de los productos. Aquí lo más importante es conocer la generación de los costos para obtener el mayor beneficio posible de ellos, minimizando todos los factores que no añadan valor.

En efecto, la contabilidad estratégica, cuenta con elementos importantes, como lo son; el Balanced Scorecard, Valor Económico Agregado (EVA), el Costeo ABC, entre otros, los cuales prestan apoyo, como soporte o herramientas de comandos integrales, para los análisis respectivos tanto interno como externos, que deben hacer las organizaciones.

\section{Ventajas Competitivas}

Señala, Torreblanca (2017), las ventajas competitivas, son aquellas que les otorgan valores diferenciales a una organización. En otras palabras, es lo que posee diferente una empresa respecto a otras competidoras, haciéndolas en muchos casos únicas, le permite mantenerse en el tiempo al ser netamente superiores a las competencias y es aplicable a varias situaciones del mercado, rentable para la empresa y costeable para el cliente.

De igual manera, Navarro (2007), afirma que es una característica de diferencia de un producto, servicio o empresa con respecto a sus competidores, esta característica llamada también ventaja tiene que ser única, diferencial, estratégicamente valorada por el mercado, y sobre todo comunicada.

Según Moreno (2008), una ventaja competitiva, es tomar aquello que la compañía hace realmente bien para así, superar a la competencia, además de darle a los clientes un beneficio, la competencia no pueda ofrecer, dándole a los consumidores una razón para elegir un producto en lugar de otro una y otra vez. 
En efecto, las ventajas competitivas, serian aquellas características, que cualquier empresa u organización, quisiera tener o poseer, con ellas, marcaria las pausas en sus sectores, tomado posesión del mercado, haciendo en muchos casos, que sea el propio mercado el cual se adapté a sus exigencias, en este sentido, existen numerosas organizaciones que han decidido cambiar el mercado, lográndolo al tener esa ventaja competitiva.

\section{Ventajas competitivas de la contabilidad estratégica}

Según Pacocruz Asesoría (2015), resalta que la contabilidad de gestión, también llamada contabilidad estratégica, puede jugar un papel muy importante en la mejora eficiente y los resultados de los diferentes procesos empresariales. Aun así, como no es obligatoria, a menudo se tiende a pensar que los beneficios aportados son poco importantes o de poco valor. Son muchas las empresas que todavía no tienen un modelo establecido de contabilidad de gestión, por considerar las mejoras que aporta son menores a los gastos de recursos que supone

Por eso es importante señalar, continua el autor, que los beneficios agregan mejoras que pueden suponer optimización en las prácticas empresariales: En consecuencia, el beneficio principal de la contabilidad directiva es que ayuda a la empresa a obtener información financiera y no financiera, clasificarla, ordenarla, presentarla, para mejorar la toma de decisiones, el control, planificación y gestión en general.

De igual manera, es una herramienta, que mejora la gestión de costos, agiliza mucho la clasificación de gastos, pero además, también permite detallar el tipo de gastos en concreto. Una buena gestión de los costos, también redundará en un mejor control del cumplimento del presupuesto y la obtención de ventajas competitivas.

Por otra parte, Farfán (2010), afirma que la contabilidad de dirección estratégica sirve de eficaz apoyo a la obtención de la excelencia empresarial, debido a la información estratégica que suministra a la Alta Dirección. Esta información no sólo sirve para fundamentar la elección de estrategias, sino también para mejorar la integración y eficiencia de los recursos humanos. Por ejemplo, en los sistemas contables de Japón se emplean más para motivar a los empleados a actuar de acuerdo con las estrategias, para suministrar datos precisos sobre costos, ingresos, beneficios; es decir, el rol contable se orienta más a influir a informar.

Las empresas creativas, continúa el autor, que tienden a la mejora continua, son aquellas donde el personal está más motivado y se siente realmente parte de ellas, aportando nuevas ideas en la mejora del negocio. En consecuencia, la consideración hacia las personas se convierte en el eje principal la cultura organizacional.

De igual manera, el principal interés en este tema se refiere al rol de la contabilidad de dirección estratégica en su labor de apoyo a la formulación de las mencionadas estrategias; en esta 
perspectiva se puede definir la contabilidad estratégica como un conjunto integrado de acciones encaminadas a asegurar una ventaja competitiva sostenible.

En consecuencia, se puede inferir, que la contabilidad estratégica, se forma como una alternativa significativa y asertiva, siendo el resultado de la unión de factores totalmente estratégicos, concatenando esfuerzos y tareas, con el manejo de información relevante, la cual será descrita y analizada por la alta gerencia, directores, para las diferentes toma de decisiones a que hubiera lugar, conllevando alcanzar ese fin último.

\section{Análisis}

Partiendo de los supuestos anteriores, donde se analizó la contabilidad estratégica como una disciplina para el alcance de ventajas competitivas, en la cual se definieron los conceptos planificación estratégica, contabilidad estratégica, estableciendo correlaciones, posturas, objetivos, elementos, y ventajas, como también el concepto de ventajas competitivas

En este sentido, se logró establecer que la contabilidad estratégica, es una variable muy importante, además de fundamental para el logro de metas, cuenta con objetivos muy bien marcados, integrada por procesos administrativos, financieros y de costos, que la fortalecen, adicionalmente soportada por elementos como el Balanced Scorecard, Valor Económico Agregado (EVA), el Costeo $\mathrm{ABC}$, entre otros, convirtiéndola sin lugar a dudas, en una verdadera disciplina para alcanzar ventajas competitivas.

\section{Conclusiones}

- Una vez analizado, el efecto que pudiese tener la contabilidad estratégica como una disciplina para el alcance de ventajas competitivas e impulsar el desarrollo socioeconómico, estructurado las definiciones, los conceptos, estableciendo correlaciones, posturas, objetivos, elementos, y ventajas de la contabilidad estratégica, como también el concepto de ventajas competitivas, se expresan las siguientes consideraciones:

- La contabilidad estratégica, se puede considerar con un elemento indispensable, que debe ser integrado a todo plan estratégico, como un factor de apoyo, para el análisis tanto interno como externo, de cualquier empresa, ayudándola a tomar decisiones, direccionándola a posicionarse competitivamente.

- De igual manera, la contabilidad estratégica, posee unos objetivos bien marcados, como lo son: proveer, analizar información para la formulación de estrategias del negocio, comunicar la estrategia en la organización mediante el establecimiento indicadores de medición del desempeño, monitorear la validez de la estrategia del negocio y todos aquellos que vayan ligados a la misión, visión y valores empresariales

- Al mismo tiempo, la contabilidad estratégica, cuenta con elementos que por separado se han establecido como herramientas de apoyo importantes, para el manejo integral de los diferentes factores que conviven dentro y fuera de la organización, como lo son el Balanced Scorecard, Valor Económico Agregado (EVA), el Costeo ABC, entre otros. 
- En consecuencia, tanto el Balanced Scorecard, Valor Económico Agregado (EVA) y el Costeo ABC, han sido clasificados como elementos de apoyo, al momento de efectuar evaluaciones profundas a determinadas áreas, con el fin de conocer sus realidades, como ellas se están comportando o manejando en sus procesos, y así determinar operaciones o estrategias, para un mejor desempeño.

- Finalmente, por todas estas razones, se puede establecer que la contabilidad estratégica, es una herramienta fundamental, debe ser incluida, tomada muy en cuenta para toda planificación estratégica, ya que de ella, se genera información importante y confidencial, al ser analizada, interpretada, puesta en ejecución, se puede logran ventajas competitivas e impulsar el desarrollo socioeconómico.

\section{Referencias Bibliográficas.}

Alcarria, J. (2008). Contabilidad financiera I. Publicacions de la Universitat Jaume I. Castellón de la Plana. España.

Arrarte, R. (2012). Contabilidad Estratégica en la gestión de empresas textiles Peruanas. Documento en línea en: file:///C:/Users/Usuario/Downloads/CONTABILIDAD_ESTRATEGICA_EN_LA_G ESTION_DE_EMPRESAS.pdf Consultada 27/06/2018.

Farfán, M. (2010). La contabilidad de direccionamiento estratégico como oportunidad de desarrollo empresarial en las Pymes. http://www.scielo.org.co/scielo.php?script=sci_arttext\&pid=S012314722010000300002 Consultado 27/06/2018.

Horngren, C; Sundem, G; Stratton William, O. (2006). Contabilidad administrativa, Pearson Educación. México.

Horngren, C; Sundem, G; Stratton William, O. (2010). Contabilidad. Documento en línea en:

http://www.facetvirtual.unt.edu.ar/pluginfile.php/28090/mod_resource/content/0/CO NTABILIDAD_hongren_harrison_oliver_2010-pdf_smallpdf.com_.pdf Consultada $28 / 06 / 2018$

Horngren, C; Foster, G; Datar, S (2017). Contabilidad de costos: un enfoque gerencial. Pearson Educación. México.

Lunar, C. (2016). Contabilidad Estratégica. Documento en línea en: http://carmelita.info/aceptas-el-reto/contabilidad-estrategica/ consultada 27/06/2018.

Martínez, F. (2002). Planeación Estratégica Creativa. Editorial PAC. México. 
Mallo, C. y Pulido, A. (2008). Contabilidad financiera. Un enfoque actual. Editorial Paraninfo. Madrid España.

Montes, C. (2009). Administración de Riesgos Empresariales. Armenia: Editorial Universitaria de Colombia.

Moreno, A. (2008). Responsabilidad Social y Corporativa: Una visión desde la empresa. Documento en línea

en: http://wwwgva.es/c_economia/web/rveh/pdfs/n12/debate1_12pdf. consultada $27 / 06 / 2018$

Navarro, L. (2007). Ventajas Competitivas dentro de la empresa. Documento en línea en: http://www.lafecha.net/articulos/empresas/ventajas-competitivas-dentro-de-laempresa/vestigacion. Consultada 27/06/2018

Pacocruz Asesoría (2015). Beneficios de la contabilidad de gestión para tu empresa. Documento en línea en: http://www.pacocruz.com/los-beneficios-de-la-contabilidadde-gestion-para-tu-empresa/ Consultada 28/06/2018.

Pelekais, C; El Kadi, O; Seijo, C; Neuman, N (2015). El ABC de la investigación. Guía didáctica. Ediciones Astro Data S.A. Maracaibo. Venezuela.

Quintal, A. (2005). Desarrollo estratégico de la pequeña empresa impulso a la economía de Yucatán. UADY. México.

Robles, G; Alcerreca C. (2008). Administración: un enfoque interdisciplinario. Pearson Educación. México.

Rodríguez, J. (2005). Cómo aplicar la planeación estratégica a la pequeña y mediana empresa. ( $5^{\mathrm{a}}$ ed). Thomson. México

SUMMA ENTERPRISSE (2016). Contabilidad Estratégica. Documento en línea: https://summaenterprisse.weebly.com/contabilidad-estrateacutegica.html Consultada 27/06/2018.

Torreblanca, F. (2017). Cómo definir la ventaja competitiva según Michael Porter Documento en línea en: https://franciscotorreblanca.es/como-definir-la-ventajacompetitiva/ consultada 27/06/2018.

Velastegui, W. (2012). Contabilidad estratégica. Documento en línea en: https://es.slideshare.net/wilsonvelas/contabilidad-estrategica consultada 27/06/2018 


\section{PARA CITAR EL ARTÍCULO INDEXADO.}

Elizalde Marín, L., \& Morales Noriega, A. (2019). Contabilidad estratégica como disciplina para alcanzar ventajas competitivas e impulsar el desarrollo socioeconómico. Ciencia Digital, 3(3.3), 241253. https://doi.org/10.33262/cienciadigital.v3i3.3.812

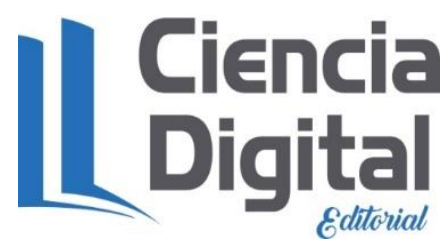

El artículo que se publica es de exclusiva responsabilidad de los autores y no necesariamente reflejan el pensamiento de la Revista Ciencia Digital.

El artículo queda en propiedad de la revista y, por tanto, su publicación parcial y/o total en otro medio tiene que ser autorizado por el director de la Revista Ciencia Digital.
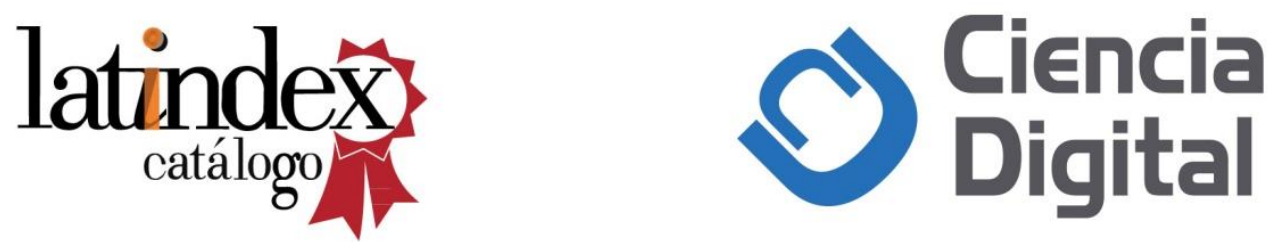